Originalien

Z Rheumatol 2021 $\cdot 80: 418-424$ https://doi.org/10.1007/s00393-021-00976-7 Angenommen: 8. Februar 2021 Online publiziert: 11. März 2021

(c) Der/die Autor(en) 2021

\section{Redaktion}

U. Müller-Ladner, Bad Nauheim

U. Lange, Bad Nauheim

Elizabeth Sierocinski ${ }^{1}$ (D) Aniela Angelow ${ }^{1}$ (D) - Armin Mainz ${ }^{2} \cdot$ Jochen Walker $^{3}$ (D) Jean-François Chenot ${ }^{1} \mathbb{B}$

'Abteilung Allgemeinmedizin, Institut für Community Medicine, Universitätsmedizin Greifswald, Greifswald, Deutschland

${ }^{2}$ Hausarztpraxis Korbach, Korbach, Deutschland

${ }^{3}$ InGef - Institut für angewandte Gesundheitsforschung Berlin (früher Health Risk Institute), Berlin, Deutschland

\title{
Patientensicherheit in der Rheumatherapie
}

\section{Labormonitoring bei Methotrexat}

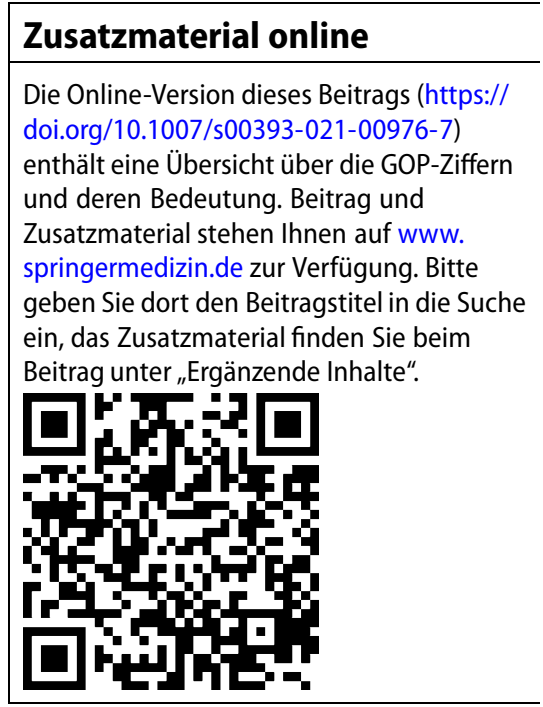

\begin{abstract}
Methotrexat (MTX) wird in der Behandlung rheumatologischer Erkrankungen häufig verwendet. Aufgrund der Möglichkeit zahlreicher Nebenwirkungen, darunter Hepatotoxizität und die potenziell tödliche Myelotoxizität, wird ein regelmäßiges Labormonitoring empfohlen. Zudem wird eine prophylaktische Folsäuregabe empfohlen. Diese Arbeit untersucht, ob die empfohlenen Laborkontrollen und Folsäureverordnungen während der MTX-Therapie nach Empfehlungen stattfinden.
\end{abstract}

\section{Hintergrund und Fragestellung}

Methotrexat (MTX) ist ein krankheitsmodifizierendes Antirheumatikum (DMARD, „disease-modifying antirheumatic drug") und in Kombination mit einer kurzfristigen Glukokortikoidtherapie Mittel der ersten Wahl in der Behandlung der rheumatoiden Arthritis (RA) [1]. MTX wird von allen synthetischen DMARDs am häufigsten verordnet [2].

Etwa drei Viertel aller MTX-therapierten RA-Patienten erleiden unerwünschte Arzneimittelwirkungen(UAW) [3]. Dies führt in 10-37\% der Fälle zu einem dauerhaften Therapieabbruch [3-5]. UAW wie gastrointestinale und Lebertoxizität sind zwar häufig, aber meist nicht lebensgefährlich. Myelotoxizität sowie die seltene Pneumonitis können lang anhaltende Schädigungen, Behinderungen oder sogar einen vorzeitigen Tod herbeiführen [3, 6-9]. Die Myelotoxizität zeigt sich als besonders gefährlich, indem sie den häufigsten Grund für eine Krankenhauseinweisung zur Behandlung einer MTX-Toxizität (78,5\%) darstellt und eine hohe Mortalitätsrate von $25 \%$ mit sich bringt [7]. Ursächlich sind dabei meist akzidentelle Überdosierungen oder toxische Akkumulationen z. B. bei Nierenversagen. Die Folsäuregabe einen Tag nach der MTXGabe verringert zwar gastrointestinale und lebertoxische UAW sowie Therapieabbrüche, aber eliminiert diese nicht vollständig [10].
Um die Zeichen einer MTX-Toxizität möglichst früh zu erkennen und Komplikationen vorzubeugen, empfehlen Experten aus deutschen sowie internationalen Fachgesellschaften ein Monitoring durch regelmäßige Kontrollen des Blutbildes, der Leberwerte und der Nierenretentionsparameter [11-17]. Die Verordnung von Folsäure und eine regelmäßige Betreuung durch einen Facharzt für Rheumatologie werden ebenfalls empfohlen.

Ziel unserer Studie war, durch eine retrospektive Analyse, basierend auf Abrechnungsdaten der gesetzlichen Krankenkasse, zu prüfen, ob das MTX-Monitoring gemäß den Handlungsempfehlungen von der Deutschen Gesellschaft für Rheumatologie (DGRh) und der Deutschen Gesellschaft für Allgemeinmedizin (DEGAM) durchgeführt wird [11, 12]. Zusätzlich wurde die Häufigkeit der Kodierung von potenziell MTX-assoziierten Komplikationen aplastische Anämie und Leberversagen sowie vom Toxizität-

\begin{tabular}{|c|c|}
\hline \multicolumn{2}{|c|}{ Abkürzungen } \\
\hline ATC & $\begin{array}{l}\text { Anatomisch-therapeutisch- } \\
\text { chemisches Klassifikationssystem }\end{array}$ \\
\hline$E B M$ & Einheitlicher Bewertungsmaßstab \\
\hline GKV & Gesetzliche Krankenversicherung \\
\hline GOP & Gebührenordnungsposition \\
\hline$I C D$ & $\begin{array}{l}\text { Internationale statistische } \\
\text { Klassifikation der Krankheiten und } \\
\text { verwandter Gesundheitsprobleme }\end{array}$ \\
\hline
\end{tabular}




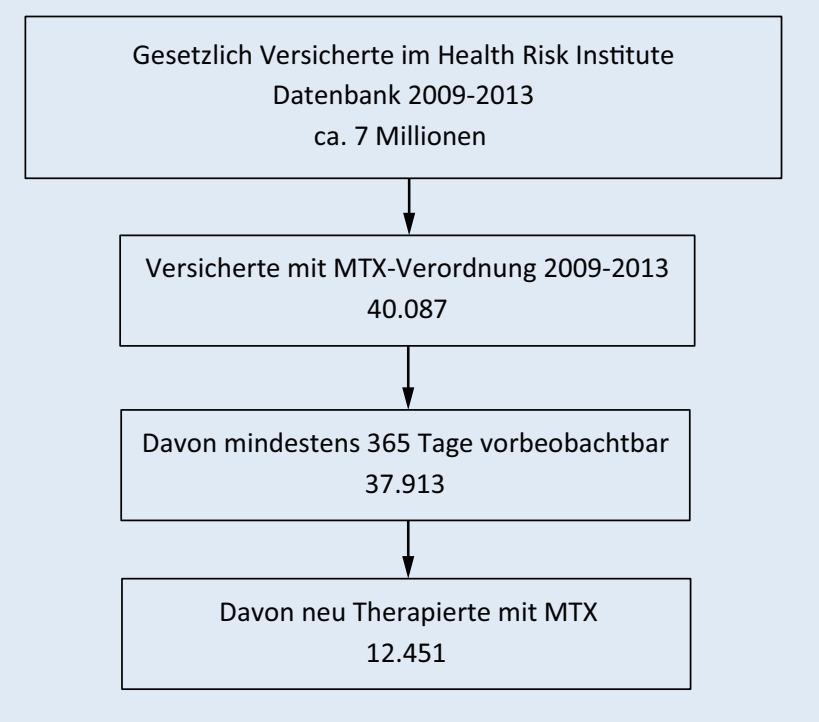

begünstigenden akuten Nierenversagen untersucht.

\section{Studiendesign und Unter- suchungsmethoden}

\section{Stichprobe}

Die retrospektive Analyse basierte auf anonymisierten Abrechnungsdaten der gesetzlichen Krankenkassen. Aus der Forschungsdatenbank des InGef (früher Health Risk Institute, ca. 7 Mio. Versicherte) wurden Erwachsene mit mindestens 2 MTX-Verordnungen (ATC-Code L01BA01) im Zeitraum von 01.01.2009 bis 31.12.2013 extrahiert [18]. Die Nutzung der Datenbank erfolgte gemäß $\$ 284$ SGB V (Sozialdaten bei den Krankenkassen) in Verbindung mit $\$ 70$ SGB V (Qualität, Humanität und Wirtschaftlichkeit). Eingeschlossen in die Analyse wurden erwachsene Patienten mit einer kodierten rheumatischen Erkrankung (ICD-10-GM M05-M18; Arthritiden und entzündliche Polyarthropathien), keiner Karzinomdiagnose (ICD-10-GM C00-C75) und keiner MTX-Verordnung $\geq 12$ Monate vor der Erstverordnung (Neuverordnung) (• Abb. 1). Daten wurden bis zu einer MTX-Therapielücke von $\geq 90$ Tagen nach Ende der Verordnungsreichweite erhoben.

\section{Monitoringparameter}

Unter der Annahme, dass das Monitoring zumindest zum Teil durch Hausärzte durchgeführt wird, wurden die Gebührenordnungspositionen (GOPs, Anhang 1, s. elektronisches Zusatzmaterial online) für die Bestimmung der von der Deutschen Gesellschaft für Allgemeinmedizin (DEGAM) empfohlenen Laboruntersuchungen genutzt (diese Empfehlungen stimmten weitgehend mit denen der Deutschen Gesellschaft für Rheumatologie [DGRh] überein; - Tab. 1). Die Häufigkeit der rheumatologischen $\mathrm{Be}$ treuung wurde ebenfalls mittels GOPs untersucht. Mittels ATC-Code (B03BB) wurde die von der DGRh empfohlene Verordnung für prophylaktische Folsäure gemäß Anlage 1 der Arzneimittelrichtlinie untersucht [19]. Zusätzlich wurden die ICD-10-GM-Codes für Ereignisse bestimmt, die potenziell auf MTX zurückzuführen sind (aplastische Anämie ICD D61.1*, Leberversagen ICD K71.1) oder die MTX-Toxizität begünstigen könnten (akutes Nierenversagen ICD N17.*).

\section{Analysen}

Die Abrechnungsdaten wurden deskriptiv ausgewertet. Anhand der Häufigkeit der Kodierungen für die empfohlenen Maßnahmen wurde analysiert, welcher Patientenanteil über die gesamte Therapiedauer gemäß der DEGAM-S1-Leitli- nie (Handlungsempfehlungen nach Klassifikation der Arbeitsgemeinschaft der wissenschaftlichen medizinischen Fachgesellschaften [AWMF]) untersucht wurde. Die Angaben beziehen sich immer auf die zu dem Zeitpunkt beobachtbaren Patienten. Die Häufigkeit der Komplikationen wurde durch Inzidenzraten von Fällen pro 1000 Personenjahre berechnet. Die Auswertungen erfolgten mit R 3.2.1.

\section{Ergebnisse}

\section{MTX-Verordnungen}

Insgesamt 12.451 Patienten begannen eine neue MTX-Therapie zwischen 2009 und 2013. Die Zahl der beobachtbaren Patienten nahm in jedem Quartal ab. Nach einem Jahr erhielt nur noch ca. die Hälfte ( $n=5914)$ eine MTX-Verordnung. Das mittlere Alter der Patienten betrug 53,8 Jahre $( \pm$ SD 14,1$)$, und $64 \%$ waren Frauen (•Tab. 2). Die mittlere Therapiedauer betrug 476 Tage.

\section{Labormonitoring}

Die von der DEGAM empfohlenen Laborkontrollen (im ersten Behandlungsmonat wöchentlich, in den 2. und 3. Monaten alle 2 Wochen und danach 1-mal in Quartal) wurden während der gesamten Therapiedauer bei $46 \%$ der Patienten für das Blutbild, $42 \%$ für die GGT, $42 \%$ für die GPT, $43 \%$ für das Kreatinin und $14 \%$ für den Urinstatus abgerechnet (•Abb. 2). Eine rheumatologische Betreuung wurde in $84 \%$ der Fälle jährlich abgerechnet. Die von der DGRh empfohlene Folsäureverordnung lag bei $74 \%$ der Patienten vor.

Die Durchführung der empfohlenen Laborkontrollen erfolgte seltener als empfohlen insbesondere zu Beginn der Therapie mit einer Bestimmung von Blutbild in 30,1\% der Patienten, von der GGT in $27,7 \%$, von der GPT in $28,3 \%$, von Kreatinin in $28,8 \%$ und Urinstatus in $8,4 \%$ in den ersten 7 Therapietagen. In dem Zeitraum zwischen dem 91. und 180. Therapietag betrug der untersuchte Patientenanteil $69,6 \%$ für das Blutbild, $65,7 \%$ für die GGT, 65,0 \% für die GPT, 
Z Rheumatol 2021 · 80:418-424 https://doi.org/10.1007/s00393-021-00976-7

(c) Der/die Autor(en) 2021

E. Sierocinski · A. Angelow · A. Mainz · J. Walker · J.-F. Chenot

\section{Patientensicherheit in der Rheumatherapie. Labormonitoring bei Methotrexat}

\section{Zusammenfassung}

Hintergrund. Methotrexat (MTX) ist das das am häufigsten verordnete krankheitsmodifizierende Antirheumatikum. Ein regelmäßiges Labormonitoring wird empfohlen, um Nebenwirkungen wie Hepatotoxizität und Myelotoxizität sowie MTX-Toxizitätbegünstigende Zustände wie eingeschränkte Nierenfunktion früh zu erkennen. Zudem wird eine prophylaktische Folsäuregabe empfohlen. Diese Arbeit untersucht, ob die empfohlene Kontrolluntersuchungen und Folsäureverordnungen während der MTXTherapie durchgeführt werden.

Material und Methoden. Abrechnungsdaten der gesetzlichen Krankenkassen vom 01.01.2009 bis 31.12.2013 wurden analysiert. Aus der Forschungsdatenbank des InGef (Institut für angewandte Gesundheitsforschung Berlin, früher Health Risk Institute) wurden 40.087 Erwachsene mit einer kodierten rheumatischen Erkrankung (ICD-10Codes M05-M18), ohne Karzinomdiagnose und ohne MTX-Verordnung $\geq 12$ Monate vor Erstverordnung extrahiert. Es wurde analysiert, ob Laborkontrollen entsprechend den Handlungsempfehlungen, eine jährliche rheumatologische Betreuung sowie die Verordnung von Folsäure erfolgten. Ergebnisse. Es begannen 12.451 Patienten eine neue MTX-Therapie im Beobachtungszeitraum. Das Blutbild, die Leberwerte und die Nierenfunktion wurden bei $42-46 \%$ und der Urinstatus bei $14 \%$ der Patienten wie empfohlen untersucht; $84 \%$ befanden sich in regelmäßiger rheumatologischer Betreuung, und $74 \%$ bekamen eine Folsäureprophylaxe. Möglicherweise MTX-assoziierte schwerwie- gende Komplikationen wurden in 0,7 bis 3,5 Fällen/1000 Personenjahre beobachtet. Diskussion. Kontrolluntersuchungen bei MTX-Therapie werden seltener als empfohlen durchgeführt. Möglicherweise MTX-assoziierte Komplikationen sind aus der Praxisperspektive sehr selten. Einerseits sind Maßnahmen für die bessere Koordination der Kontrolluntersuchungen erforderlich. Andererseits müssen der Nutzen des Monitorings und die Abstände der Monitoringintervalle durch empirische Untersuchungen besser belegt werden.

Schlüsselwörter

Pharmakovigilanz $\cdot$ Medikamentenmonitoring · Methotrexat · Unerwünschte Arzneimittelwirkungen $\cdot$ Handlungsempfehlungen

\section{Patient safety in the treatment of rheumatic diseases. Laboratory monitoring in methotrexate treatment}

\section{Abstract}

Background. Methotrexate (MTX) is the most commonly prescribed disease-modifying drug in the treatment of rheumatic diseases. Regular laboratory testing is recommended to recognize side effects, such as hepatotoxicity and myelotoxicity as well as decreases in renal function that may cause toxic MTX accumulation. Additionally, folic acid is recommended as prophylaxis against specific side effects. In this study we investigated whether laboratory monitoring and prescription of folic acid took place according to published recommendations.

Material and methods. Claims data from the statutory health insurance from 1 January 2009 to 31 December 2013 were retrospectively analyzed. A total of 40,087 adults with a rheumatic diagnosis (ICD10 codes M05-M18), no malignant disease and no previous MTX prescription within 12 months were extracted from the InGef (Institute for Applied Health Research in Berlin, formerly Health Risk Institute) research database. The frequency of recommended laboratory testing, appointments with rheumatologists and the prescription of folic acid prophylaxis were investigated. Results. Of the patients 12,451 began treatment with MTX in the observation period. Between $42 \%$ and $46 \%$ of recommended blood counts, liver values and kidney function tests and $14 \%$ of urinalyses were performed according to recommendations. Of the patients $84 \%$ were seen regularly by a rheumatologist and $74 \%$ received a prescription for prophylactic folic acid. Serious conditions potentially resulting from MTX treatment were observed in $0.7-3.5$ cases $/ 1000$ person years. Discussion. Laboratory monitoring in the context of MTX treatment is carried out less frequently than recommended in the literature. Potential MTX-associated serious complications are rare from a practice perspective. On the one hand solutions are needed for a better coordination of laboratory monitoring. On the other hand more empirical evidence is needed regarding the benefits of laboratory monitoring and the appropriate intervals thereof.

Keywords

Pharmacovigilance - Drug monitoring . Methotrexate · Adverse events · Guidelines
$67,2 \%$ für Kreatinin und 27,7\% für den Urinstatus (• Abb. 3).

\section{Potenzielle Komplikationen und UAW-begünstigende Zustände}

Akutes Nierenversagen wurde in 3,5 Fällen/1000 Personenjahre (0,3\%), Leberversagen in 0,7 Fällen/1000 Personenjahre $(0,1 \%)$ und aplastische An- ämie in 0,9 Fällen/1000 Personenjahre $(0,1 \%)$ in den Abrechnungsdaten kodiert (•Tab. 3).

\section{Diskussion}

\section{Zusammenfassung}

Die Kontrolluntersuchungen bei MTXTherapie wurden seltener durchgeführt, als in der DEGAM-Leitlinie empfohlen. Abweichungen von den Empfehlungen zeigten sich insbesondere zu Beginn der Therapie. Bei knapp drei Viertel der Patienten wurde Folsäure verordnet, und mehr als $80 \%$ der Patienten befanden sich in rheumatologischer Betreuung. Potenziell auf MTX zurückführbare Komplikationen und Toxizität-begünstigendes 
Tab. 1 Empfohlene Maßnahmen in der MTX-Therapie nach der S1-DEGAM-Leitlinie „Medikamentenmonitoring" und der Deutschen Gesellschaft für Rheumatologie e.V.

\begin{tabular}{l} 
Gesellschaft \\
\hline Deutsche Gesellschaft \\
für Rheumatologie \\
e. V. $(D G R h)^{a}$
\end{tabular}

Maßnahmen

Blutbild einschließlich Thrombozyten und Differenzialblutbild, GOT, GPT, alkalische Phosphatase, Kreatinin

\section{Häufigkeit}

Vor Therapiebeginn

Nach 1 bis 2 Wochen

Dann nach weiteren 2 bis

3 Wochen

Danach alle 4 Wochen

Deutsche Gesellschaft für Allgemeinmedizin und Familienmedizin (DEGAM)
Befragung und klinische Untersuchung: Exanthem, Stomatitis, gastrointestinale Symptome, Fieber, Luftnot, Husten, Blutungen

Folsäure $5 \mathrm{mg}$

$\mathrm{BB}, \mathrm{GGT}, \mathrm{GPT}$, Kreatinin, Urinstatus

Bei längerer komplikationsloser Verträglichkeit weitere Streckung auf 6 bis 12 Wochen möglich $^{b}$

Einen Tag nach der MTX-Gabe Im ersten Monat: wöchentlich Im 2. und 3. Monat: alle 2 Wochen

Ab dem 4. Monat: 1-mal im Quartal

BB Blutbild, GGT Gamma-Glutamyltransferase, GPT Glutamat-Pyruvat-Transaminase, eGFR Kreatinin (Nierenfunktion), Urinstatus: Diagnostik mittels Urinteststreifen

'Die Empfehlungen sind fast identisch mit Expertenempfehlungen aus 2009 (Tarner et al. 2009) [13] ${ }^{b}$ Beibehalten der engmaschigen Kontrolle wird bei älteren Patienten mit chronischen Erkrankungen empfohlen

Tab. 3 Auftreten potenziell MTX-bedingter Komplikationen bzw. Toxizität-begünstigender Zustände während der Beobachtungszeit (2009-2013)

\begin{tabular}{lll} 
Komplikation & Fälle/1000 Personenjahre & $\mathbf{9 5} \% \mathbf{- C l}$ \\
\hline Akutes Nierenversagen & 3,48 & $2,64-4,51$ \\
Leberversagen & 0,68 & $0,34-1,22$ \\
\hline Aplastische Anämie & 0,93 & $0,52-1,53$
\end{tabular}

akutes Nierenversagen konnten beobachtet werden.

\section{Notwendigkeit des Monitorings}

Unsere Daten weisen auf ein selteneres Monitoring als in den Empfehlungen der DEGAM, der DGRh und internationaler Gesellschaften angegeben. Betont in diesen Empfehlungen werden mindestens 4-wöchentliche Laborkontrollen am Anfang der MTX-Therapie [11, 12], da UAW wie Leberschädigungen und Myelotoxizität häufiger in den ersten Monaten der Therapie auftreten [3]. Nur bei längerer komplikationsloser Verträglichkeit der MTX-Therapie sollten seltenere Kontrollen alle 6 bis 12 Wochen in Erwägung gezogen werden [11, 12, 14-17]. Es besteht ein Risiko für UAW für die gesamte Therapiedauer [8]: Leberwerterhöhungen bis hin zu schwerer Lebertoxizität treten in bis zu einem Drittel der MTXTherapierten auf $[5,20]$. Die seltene $(5 \%)$, aber tödliche (25\% Mortalitätsrate bei hospitalisierten Patienten) Myelotoxizi- tät kann durch einen erhöhten MTXSpiegel aufgrund nur leichter, passagerer Einschränkung der Nierenfunktion, bei Interaktionen mit anderen Medikamenten oder Fehldosierungen (z. B. versehentliche tägliche, nicht wöchentliche Gabe) auftreten $[5,6]$. In unserer Studie beobachteten wir Ereignisse, die potenzielle Komplikationen einer MTX-Therapie darstellten (Leberversagen und aplastische Anämie) oder eine toxische Erhöhung des MTX-Spiegels verursachen konnten (akutes Nierenversagen). Das Auftreten dieser Ereignisse, bei denen eine Implikation von MTX erwogen wird, kann eine Begründung für ein langfristiges Monitoring sein. Allerdings gibt es keine empirische Datengrundlage für optimale Monitoringintervalle. Die Empfehlungen basieren lediglich auf Expertenkonsens.

Eine französische Studie zeigt ebenfalls ein selteneres MTX-Monitoring, als in Handlungsempfehlungen dargestellt [21]. In dieser Studie werden häufigere Kontrolluntersuchungen mit frühe-
Tab. 2 Demografie der Versicherten mit neuer MTX-Therapie zwischen 2009 und $2013(N=12.451)$

\begin{tabular}{|l|l|}
\hline Mittleres Alter in Jahren (SD) & $53,8( \pm 14,1)$ \\
\hline Minimum Alter & 18 \\
Maximum Alter & 95 \\
Anteil Frauen & $64,0 \%$
\end{tabular}

ren MTX-Therapieabbrüchen assoziiert [21]. Ob diese Therapieabbrüche UAW oder deren Komplikationen mindern, ist unklar. Es gibt keine Evidenz, ob die oft akut auftretenden schwerwiegenden Komplikationen überhaupt durch das Monitoring vermieden oder reduziert werden können oder ob Labormonitorings im Allgemeinen eher unnötige Eingriffe und Arbeitsaufwände für den Patienten und Arzt darstellen [22].

\section{Zugrunde liegende Faktoren}

Die Diskrepanz zwischen Handlungsempfehlungen und der Durchführung des Monitorings wurde bisher in der Literatur nicht geklärt. Fehlende Informationen, Zeit- und Ressourcenmangel sowie Unterversorgung stellen mögliche Einflussfaktoren dar. Ein hoher Anteil der Patienten in unserer Studie wurde rheumatologisch betreut. Die verfügbaren Daten erlauben es nicht, die Absprachen oder mangelnden Absprachen zur Koordination einzelner Versorgungsaufgaben zwischen Hausärzten und Rheumatologen nachzuvollziehen. Es ist möglich, dass Patienten nicht über die Notwendigkeit des Monitorings und Hausärzte über ihre Rolle als Koordinatoren des Monitorings von den MTX verordnenden Ärzten informiert werden. In Praxen fehlen möglicherweise auch die notwendige Zeit und Ressourcen, um Patienten regelmäßig für das Monitoring einzubestellen. Es ist möglich, dass mit MTX behandelte Patienten im Rahmen des Ärztemangels in Deutschland hausärztlich und rheumatologisch unterversorgt sind und deswegen keine Monitoringtermine wahrnehmen. Weiterhin könnte eine mangelnde Adhärenz unabhängig von der Versorgungslage eine Rolle spielen. 


\section{Originalien}

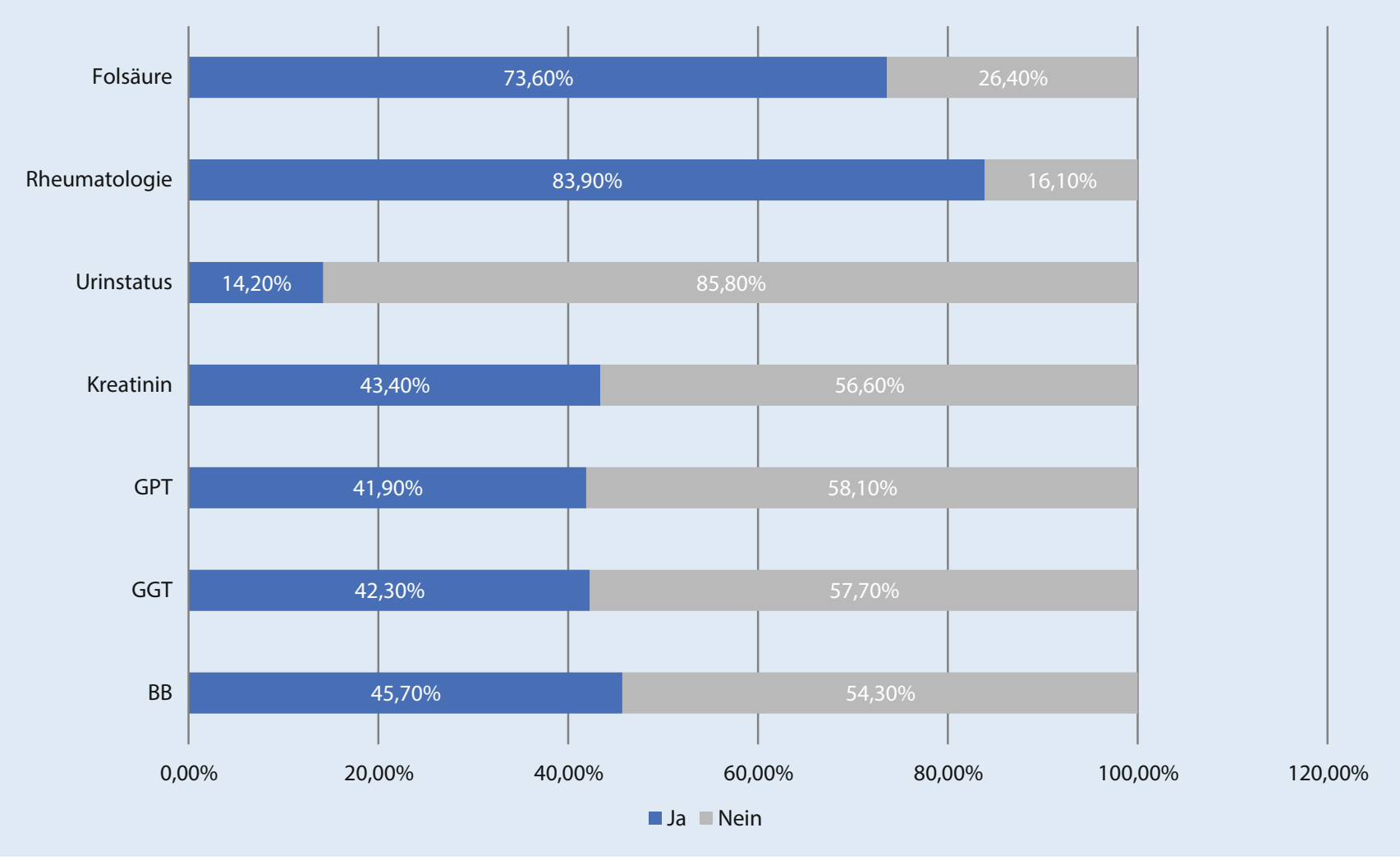

Abb. 2 А Patientenanteil mit DEGAM-empfohlenem Labormonitoring, rheumatologischer Betreuung sowie DGRh-empfohlener Folsäureverordnung über die gesamte Therapiedauer (2009-2013)

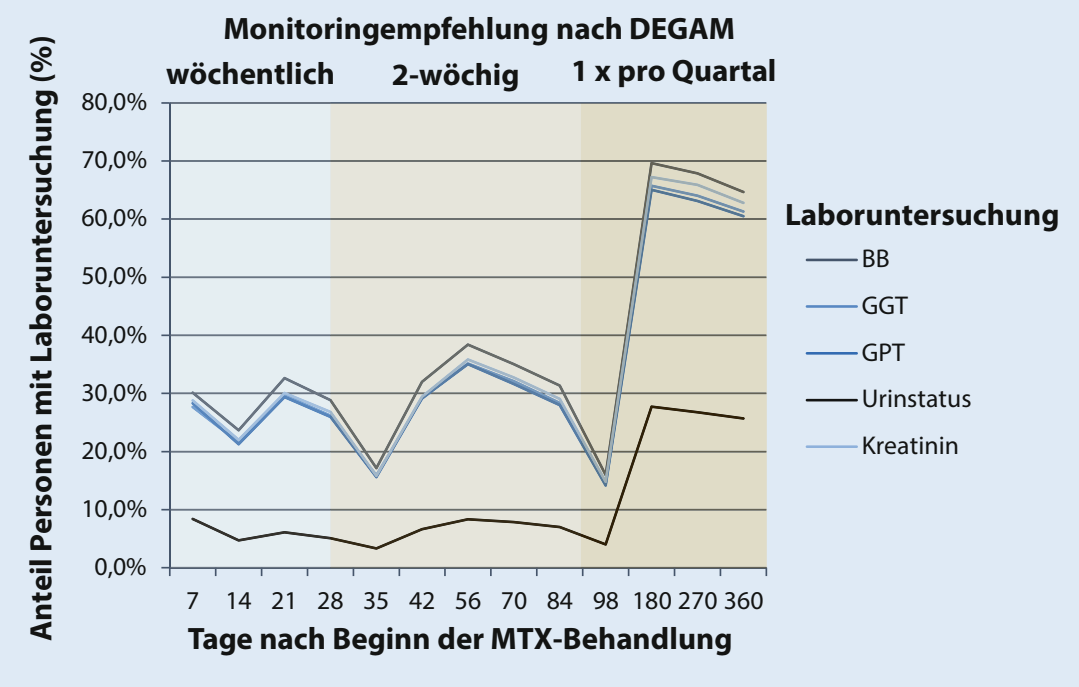

Abb. 3 A Laborbestimmungen im MTX-Monitoring (2009-2013)

\section{Potenzielle Lösungen}

Eine Methode, um Monitoringuntersuchungen in der Versorgung chronischer Erkrankungen zu etablieren, sind Disease-Management-Programme (DMP), weil diese mit einer extrabudgetären
Vergütung verknüpft sind. Das in 2005 und 2014 beim Gemeinsamen Bundesausschuss (G-BA) beantragte DMP bei Rheuma ist zugunsten anderer Volkserkrankungen verschoben worden [23]. Eine Verbesserung der direkten Kommunikation zwischen Spezialisten und
Hausärzten ist aufgrund des bereits zeitlich angespannten Praxisalltags nur eingeschränkt durchführbar. Jedoch muss die Zuständigkeit für die Kontrollen zwischen Rheumatologen und Hausärzten besser abgesprochen und koordiniert werden. Zum Beheben des Informationsdefizits könnten aktuelle Handlungsempfehlungen an Hausarztpraxen disseminiert werden. Um das Monitoring zu begünstigen und Patienten regelmäßig einzubestellen, kämen Softwarelösungen in der elektronischen Patientenakte infrage, die ein Kontrollschema beinhalten und Fachangestellte über notwendige Monitoringtermine informieren. Patienten können über die Notwendigkeit des Monitorings breitflächig in einem standardisierten Aufklärungsverfahren mittels eines Aufklärungsvideos informiert werden.

\section{Standardisierung der Empfehlungen}

Die verfügbaren Empfehlungen stimmen in den zeitlichen Abständen von Monito- 
ringuntersuchungen großenteils überein, aber die empfohlenen Kontrollparameter und Maßnahmen unterscheiden sich. Beispielsweise wird die Erhebung eines Urinstatus in der DEGAM-Leitlinie und in der MTX-Fachinformation [24] empfohlen, aber weder von der Deutschen Gesellschaft für Rheumatologie noch von anderen internationalen Gesellschaften erwähnt $[11,12]$. Dies gilt als mögliche Erklärung für die besonders niedrige Anzahl an durchgeführten Urinanalysen in unserer Studie. Andere Handlungsempfehlungen empfehlen eine Thoraxröntgenaufnahme vor Therapiebeginn, um mögliche Lungenvorerkrankungen und dabei Patienten mit erhöhtem Risiko für pulmonale UAW zu erkennen [17]. Die Notwendigkeit aller empfohlenen Untersuchungen muss überprüft werden, um unnötige Untersuchungen zu vermeiden [25]. Standardisierte Empfehlungen würden Verwirrung und Fehlinformationen minimieren und stellen eine Voraussetzung für die breitflächige Implementierung der oben genannten Lösungsstrategien dar. Eine wichtige Voraussetzung für standardisierte Monitoringempfehlungen stellt die Verbesserung der Evidenzlage bezüglich der Laborparameter und Monitoringintervalle dar. Dies stellt eine Herausforderung nicht nur für das MTX-Monitoring, sondern für das Labormonitoring chronischer Erkrankungen wie Diabetes und Bluthochdruck dar [22].

\section{Stärken und Limitationen}

Basierend auf den Abrechnungsdaten kann nicht evaluiert werden, ob die Laborbestimmungen direkt auf die MTXTherapie zurückzuführen sind. Bei den erfassten Ereignissen von Leberversagen, Nierenversagen und aplastischer Anämie mit Krankenhausaufenthalt kann von einer Vollständigkeit ausgegangen werden, allerdings muss MTX nicht kausal verantwortlich sein. Andere Aspekte des Medikamentenmonitorings wie Therapietreue, Erfassen der Verträglichkeit und Wirksamkeit auf Krankheitsaktivität können mit Abrechnungsdaten nicht abgebildet werden. Dies stellt laut DGRhTherapieüberwachungsbogen einen unerlässlichen Teil des Monitorings dar
[12]. Es ist möglich, dass Laborkontrollen und insbesondere Urinstatus nicht kodiert worden sind oder mit anderen als die in unserer Studie benutzten EBMZiffern abgerechnet worden sind. Es ist möglich, dass wir den Anteil der Patienten, die Folsäure erhalten, unterschätzen, da dies auch frei verkäuflich ist. Die Zahl der beobachtbaren Patienten nahm im Beobachtungszeitraum ständig ab. Die Gründe dafür lassen sich aus Abrechnungsdaten nicht nachvollziehen.

\section{Fazit für die Praxis}

Die Verordnung von MTX steigt jährlich. Das Labormonitoring wird deutlich seltener durchgeführt, als in Handlungsempfehlungen empfohlen wird. Weitere Studien sollen die zugrunde liegenden Faktoren ermitteln; eine Evidenzlage für standardisierte Empfehlungen ist ebenfalls notwendig. Einerseits sind Maßnahmen für die bessere Koordination der Kontrolluntersuchungen, wie z. B. Softwarelösungen oder Aufklärungsvideos für die Durchführung eines empfohlenen MTX-Labormonitorings, erforderlich, andererseits müssen der Nutzen des Monitorings und die Abstände der Monitoringintervalle durch empirische Untersuchungen besser belegt werden.

\section{Korrespondenzadresse}

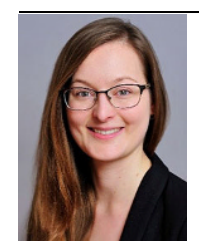

Elizabeth Sierocinski, MD

Abteilung Allgemeinmedizin, Institut für Community Medicine, Universitätsmedizin Greifswald

Fleischmannstr. 6, 17485 Greifswald, Deutschland elizabeth.sierocinski@ med.uni-greifswald.de

Danksagung. Die Autoren möchten sich bei Frau Dr. Simone Kiel für ihre sprachliche Unterstützung und inhaltliches Feedback zu dem Projekt herzlich bedanken.

Funding. Open Access funding enabled and organized by Projekt DEAL.

\section{Einhaltung ethischer Richtlinien}

Interessenkonflikt. A. Mainz ist Autor der DEGAMLeitlinie "Medikamentenmonitoring". E. Sierocinski, A. Angelow, J. Walker und J.-F. Chenot geben an, dass kein Interessenkonflikt besteht.

Für diesen Beitrag wurden von den Autoren keine Studien an Menschen oder Tieren durchgeführt.

Open Access. Dieser Artikel wird unter der Creative Commons Namensnennung 4.0 International Lizenz veröffentlicht, welche die Nutzung, Vervielfältigung, Bearbeitung, Verbreitung und Wiedergabe in jeglichem Medium und Format erlaubt, sofern Sie den/die ursprünglichen Autor(en) und die Quelle ordnungsgemäß nennen, einen Link zur Creative Commons Lizenz beifügen und angeben, ob Änderungen vorgenommen wurden.

Die in diesem Artikel enthaltenen Bilder und sonstiges Drittmaterial unterliegen ebenfalls der genannten Creative Commons Lizenz, sofern sich aus der Abbildungslegende nichts anderes ergibt. Sofern das betreffende Material nicht unter der genannten Creative Commons Lizenz steht und die betreffende Handlung nicht nach gesetzlichen Vorschriften erlaubt ist, ist für die oben aufgeführten Weiterverwendungen des Materials die Einwilligung des jeweiligen Rechteinhabers einzuholen.

Weitere Details zur Lizenz entnehmen Sie bitte der Lizenzinformation auf http://creativecommons.org/ licenses/by/4.0/deed.de.

\section{Literatur}

1. Smolen JS et al (2017) EULAR recommendations for the management of rheumatoid arthritis with synthetic and biological disease-modifying antirheumatic drugs: 2016 update. Ann Rheum Dis 76(6):960

2. Schwabe U et al (2019) Arzneiverordnungs-Report 2019

3. Salliot C, van der Heijde D (2009) Long-term safety of methotrexate monotherapy in patients with rheumatoid arthritis: a systematic literature research. Ann Rheum Dis 68(7):1100-1104

4. Lopez-Olivo MA et al (2014) Methotrexate for treating rheumatoid arthritis. Cochrane Database Syst Rev. https://doi.org/10.1002/14651858. CD000957.pub2

5. Romão VC et al (2014) Three decades of lowdose methotrexate in rheumatoid arthritis: can we predict toxicity? Immunol Res 60(2-3):289-310

6. Buschmann C et al (2007) Todesfälle nach Lowdose-Therapie mit Methotrexat. Rechtsmedizin 17(2):89-94

7. Kivity S et al (2014) Clinical characteristics and risk factors for low dose methotrexate toxicity: a cohor of 28 patients. Autoimmun Rev 13(11):1109-1113

8. Wang W, Zhou H, Liu L (2018) Side effects of methotrexate therapy for rheumatoid arthritis: a systematic review. Eur J Med Chem 158:502-516

9. Pannu AK (2019) Methotrexate overdose in clinical practice. Curr Drug Metab 20(9):714-719

10. Shea B et al (2013) Folic acid and folinic acid for reducing side effects in patients receiving methotrexate for rheumatoid arthritis. Cochrane Database Syst Rev. https://doi.org/10.1002/ 14651858.CD000951.pub2 
11. Mainz A Medikamentenmonitoring. 2013, Deutsche Gesellschaft für Allgemeinmedizin und Familienmedizin. https://www.awmf.org/leitlinien/ detail/II/053-037.html.Zugegriffen: 29.07.2020

12. Deutsche Gesellschaft für Rheumatologie (2014) Therapie mit Methotrexat. https://dgrh.de/dam/ jcr:0fff25d9-b2ea-4fca-94bc-06b03a0e09fd/ methotrexat_arzt_2014_07.pdf. Zugegriffen: 29.07.2020

13. Tarner letal (2009) Evidenzbasierte Empfehlungen einer nationalen Expertenrunde zum Einsatz von Methotrexat bei entzündlich-rheumatischen Erkrankungen. Akt Rheumatol 34:59-66

14. Chakravarty K et al (2008) BSR/BHPR guideline for disease-modifying anti-rheumatic drug (DMARD) therapy in consultation with the British Association of Dermatologists. Rheumatology 47(6):924-925

15. Singh JA et al (2016) 2015 American College of Rheumatology guideline for the treatment of rheumatoid arthritis. Arthritis Rheumatol 68(1):1-26

16. Pavy $S$ et al (2006) Methotrexate therapy for rheumatoid arthritis:clinical practiceguidelines based on published evidence and expert opinion. Joint Bone Spine 73(4):388-395

17. NHS Oxfordshire Clinical Commissioning Group (2015) Methotrexate for use in rheumatology, dermatology, neurology, gastroenterology, ophthalmology and respiratory medicine: shared care protocol

18. Andersohn F, Walker J (2016) Characteristics and external validity of the German Health Risk Institute(HRI) Database. Pharmacoepidemiol Drug Saf 25(1):106-109

19. Gemeinsamer Bundesausschuss (2018) Anlage I zum Abschnitt $F$ der Arzneimittel-Richtlinie: Gesetzliche Verordnungsausschlüsse in der Arzneimittelversorgung und zugelassene Ausnahmen

20. Conway R et al (2015) Risk of liver injury among methotrexate users: a meta-analysis of randomised controlled trials. Semin Arthritis Rheum 45(2):156-162

21. MazaudC, Fardet L (2019) Daily practices regarding safety monitoring of low-dose methotrexate and comparison to guidelines: a population-based cohort study. Therapie. https://doi.org/10.1016/j. therap.2019.05.004

22. Elwenspoek MMC et al (2019) Are guidelines for monitoring chronic disease in primary care evidence based? BMJ365:12319

23. Beerheide $R$ (2016) Versorgung: DMP bei Rheuma. Dtsch Arztebl 113(49):A-2250

24. Ratiopharm (2019) Fachinformation: MTXratiopharm ${ }^{\circledR}$ Injektionslösung in einer Fertigspritze

25. Kerr EA et al (2020) Identifying recommendations for stopping or scaling back unnecessary routine services in primary care. JAMA Intern Med. https:// doi.org/10.1001/jamainternmed.2020.4001

\section{In eigener Sache}

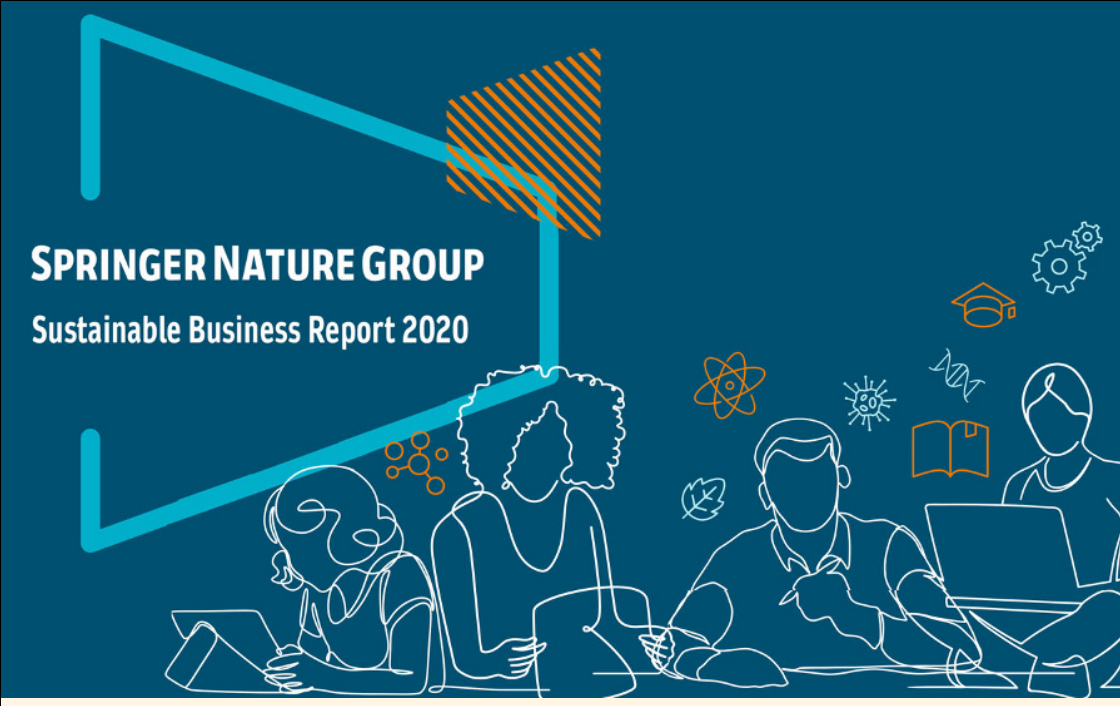

\section{Nachhaltigkeit bei Springer Nature}

Unser Sustainable Business Report 2020 zeigt, welche Fortschritte wir in den Bereichen Umwelt, Soziales und Unternehmensführung über die letzten Jahre erzielen konnten und berücksichtigt dabei auch externe Einflüsse wie die außergewöhnliche Situation im Jahr 2020 durch die COVID-19Pandemie. Als Unternehmen, das nachhaltig und verantwortungsvoll denkt und handelt wollen wir einen positiven Beitrag leisten. Verantwortung ist einer unserer Grundwerte und wir sind bestrebt, als nachhaltiges und ethisches Unternehmen zu handeln, das sich verpflichtet, die Auswirkungen seiner Tätigkeit auf die Umwelt zu berücksichtigen. Wir sind überzeugt, dass die von uns veröffentlichten Inhalte wichtige Gespräche und Diskussionen ermöglichen, die sich positiv auf Gesellschaft und Umwelt auswirken und wollen dieses Potential weiter ausbauen.

Im März 2020 haben wir uns dazu verpflichtet, klimaneutral zu werden. Bereits Ende 2020 haben wir dieses Ziel erreicht und sind - im Hinblick auf unsere Büroräumlichkeiten, Fahrzeugflotte und Geschäftsreisen - klimaneutral. Möglich wurde dies durch die erheblich gesteigerte Nutzung von Ökostrom und CO2-Ausgleichszahlungen. Als Verlag, der aktuellste Forschungsergebnisse zum Klimawandel veröffentlicht, wollen wir außerdem die Aufmerksamkeit für dieses Thema stärken - extern, aber auch intern bei rund $10.000 \mathrm{Be}$ schäftigten, die weltweit bei Springer Nature arbeiten.
Durch den Einsatz neuer Technologien wurden 2020 mehr als drei Milliarden Artikel und Buchkapitel über unsere Plattformen heruntergeladen und damit 50\% mehr als 2019. Für uns ist Technologie der Schlüssel, um Wissen schneller, nachhaltiger und kostengünstiger für noch mehr Menschen in aller Welt verfügbar zu machen.

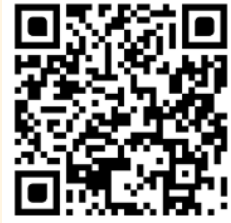

Mit dem QR-Code zur Kurzzusammenfassung des Nachhaltigkeitsreport.

Unsere Veröffentlichung im Jahr 2020:

- 3.000+Zeitschriften

- 372.000+ Forschungsartikel

- 13.000+ Neue Bücher

- 100 Neue Lehrbuchreihen, die von mehr als 30 Millionen Studierenden genutzt werden 